J3eA, Journal sur l'enseignement des sciences et technologies de l'information et des systèmes, Volume 2, Hors-Série 1, 3 (2003)

DOI : http://dx.doi.org/10.1051/bib-j3ea:2003503

(C) EDP Sciences, 2003

Commande optimale des systèmes dynamiques hybrides

C. Iung et P. Riedinger

Centre de Recherche en Automatique de Nancy, UMR 7039

Institut National Polytechnique de Lorraine - Université Henri Poincaré

ENSEM

2 av. de la Forêt de Haye

F-54516 Vandoeuvre-lès-Nancy Cedex, France 


\title{
Commande Optimale des Systèmes Dynamiques Hybrides
}

\author{
C.Iung ${ }^{*}$, P. Riedinger ${ }^{*}$
}

*Centre de Recherche en Automatique de Nancy UMR 7039

Institut National Polytechnique de Lorraine - Université Henry Poincaré

Adresse ENSEM 2, Av de la Forêt de Haye

54516 Vandoeuvre-les-Nancy Cedex

\section{Introduction}

Contrairement au cas des systèmes continus ou des systèmes à événements discrets pour lesquels la notion de commande et les problèmes de synthèse associés sont bien identifiés et clairement définis, la commande des systèmes hybrides est une notion beaucoup plus large et le domaine de recherche correspondant n'en est qu'à ses débuts. En effet, le fait que ces systèmes font intervenir deux types de dynamiques, une dynamique continue et une autre discrète, favorise la diversité des formulations du problème de commande hybride rencontrées dans la littérature

Bien que le nombre de contributions dans ce domaine ait considérablement augmenté ces dernières années, la formulation du problème de commande de systèmes hybrides reste une formulation spécifique à chaque contribution ou groupes de contributions et il est difficile de dégager une formulation unifiée de ce problème. 
On peut considérer que trois grandes classes de méthodes sont disponibles pour aborder les problèmes de commande optimale de manière générale:

- Le principe d'optimalité de Bellman qui a donné lieu à la programmation dynamique dans le cas discret et à l'équation d'Hamilton-Jacobi-Bellman (HJB) pour des systèmes continus,

- Les méthodes variationnelles qui ont trouvé leur forme achevée dans le théorème du maximum de Pontriaguine,

- Les approches heuristiques bien adaptées aux problèmes de très grande dimension avec peu de propriétés analytiques

Nous n'évoquerons pas ici les méthodes heuristiques dont on trouve des exemples dans d'autres communications. La programmation dynamique est une voie d'investigation évidente (dualité discret -continu) pour la résolution d'un problème de commande optimale hybride. Cette formulation nécessite soit une discrétisation complète du problème avec un risque d'explosion combinatoire, soit la résolution d'équations aux dérivées partielles d'HJB. Or ce problème est réputé difficile lorsque la solution est discontinue. L'utilisation du Principe du Maximum de Pontryaguine bien que moins naturelle en raison des aspects discrets, permet avec une extension de contourner cette difficulté par l'introduction d'un système adjoint et la résolution d'un système différentiel. La section 1 rappelle les quelques résultats fondamentaux de la commande optimale dans le cas continu. La section 2 précise la notion de Systèmes Dynamiques Hybrides et introduit une modélisation commode pour traiter le problème de commande. La programmation dynamique s'applique directement aux SDH (section 3), même si sa mise en œuvre pose des problèmes de complexité. On présente ensuite une extension aux SDH des résultats classiques du Principe du maximum (section 4). Un exemple illustre les deux approches évoquées et certaines difficultés inhérentes à la nature hybride des problèmes (section 5). Le lecteur pourra consulter les travaux cités en référence pour la démonstration des résultats exposés.

\section{1-Rappels de quelques résultats classiques}

\subsection{La programmation dynamique}

Le principe d'optimalité de Bellman s'applique sans hypothèses restrictives aux système dynamiques pour lesquels on cherche à minimiser un coût additif.

Le système dynamique [KAL ] est défini par:

- un espace ordonné de réels en général appelé espace des temps $T$

- un espace d'état $X$

- un espace de commandes $\Omega: T \rightarrow U$

- une fonction de transition d'état $\varphi\left(t, t_{0}, x_{0}, u\right)$ qui donne l'état à l'instant $t$ à partir de l'état initial $x_{\dot{a}}$ sous la commande $u$ 
- quelques axiomes classiques (causalité, consistance, concaténation) Le critère à minimiser est un critère additif

$$
J\left(t, t_{0}, x, u\right)=J\left(t_{1}, t_{0}, x, u\right)+J\left(t, t_{1}, x_{1}, u\right) ; \forall t_{0} \leq t_{1} \leq t ; x_{1}=\varphi\left(t_{1}, t_{0}, x, u\right)
$$

On démontre alors que le problème peut se résoudre en deux étapes. La valeur optimale du critère $V\left(t, t_{0}, x_{0}\right)=\min _{u} J\left(t, t_{0}, x_{0}, u\right)$ peut être calculée par la formule suivante :

$$
V\left(t, t_{0}, x_{0}\right)=\min _{u}\left\{J\left(t_{1}, t_{0}, x_{0}, u\right)+V\left(t, t_{1}, x_{1}\right)\right\} ; x_{1}=\varphi\left(t_{1}, t_{0}, x_{0}, u\right)
$$

Cette formule très générale conduit, avec un choix des instants intermédiaires, aux algorithmes de programmation dynamique. Lorsque $T=N$, on obtient une équation de récurrence, dans le cas continu, elle conduit à l'équation d'Hamilton Jacobi-Bellman

$$
\frac{\partial V\left(t_{f}, t, x(t)\right)}{\partial t}=-\inf _{u}\left\{L(x(t), u, t)+\left[\frac{\partial V\left(t_{f}, t, x(t)\right)}{\partial x}\right]^{T} f(x(t), u, t)\right\}
$$

Avec le critère à minimiser

$$
J=\int_{0}^{f} L(x(t), u(t), t) d t+\varphi\left(x\left(t_{f}\right), t_{f}\right)
$$

et l'équation d'évolution

$$
\dot{x}=f(x, u, t), \quad x \in \mathfrak{R}^{n}
$$

\subsection{Méthodes variationelles - théorème de Pontriaguine}

Elles s'appuient sur un développement local des solutions autour de la solution optimale. La solution optimale étant caractérisée par le fait que si l'on envisage commande 'voisine', on trouve un résultat moins bon. Envisagées aux $17^{\circ}$ siècle, les résultats sont synthétisés dans les travaux de l'école Pontriaguine et le théorème du maximum.

Soit le système dynamique

$$
\dot{x}=f(x, u, t), \quad x \in \mathfrak{R}^{n}
$$

Où $\mathrm{f}$ est continue sur $\mathrm{X} \times \mathrm{U}$ 
Le critère

$$
J=\int_{0}^{f} L(x(t), u(t), t) d t
$$

Si $\hat{x}$ et $\hat{u}$ sont optimales, alors il existe une fonction $\lambda$ et une constante $\lambda_{0}<0$, telles que :

- $\quad \mathrm{x}$ et $\lambda$ vérifient les équations canoniques de Hamilton

$$
\dot{x}=\frac{\partial H}{\partial \lambda} \quad \dot{\lambda}=-\frac{\partial H}{\partial x}
$$

- $\quad \hat{\mathrm{u}}(\mathrm{t})$ minimise la fonction hamiltonienne $\operatorname{sur}\left[\mathrm{t}_{0}, \mathrm{t}_{\mathrm{f}}\right]$

$$
H\left(\lambda_{0}, \lambda, \hat{x}, u\right)=\lambda_{0} L(\hat{x}, u)+\lambda^{T} f(\hat{x}, u)
$$

- Aux extrémités, les conditions de transversalité sont satisfaites

$$
\text { si } x\left(t_{0}\right) \in C_{0} \quad x\left(t_{f}\right) \in C_{f} \text { alors } \lambda\left(t_{0}\right) \in N_{C o} x\left(t_{0}\right) \lambda\left(t_{f}\right) \in N_{C_{f}} x\left(t_{f}\right)
$$

$N_{C_{i}} x\left(t_{i}\right)$ sont les normales aux variétés sur lesquelles évolue $\mathrm{x}$ aux extrémités

Sous des conditions assez faibles, s'il existe des commandes satisfaisant aux conditions aux limites, alors il existe une commande optimale, mais la recherche pratique des commandes optimales peut être complexe. En effet avec les conditions aux limites en $t_{0}$ et $t_{f}$ sur l'état et sur la variable adjointe $\lambda$, on à résoudre un problème de tir de dimension $2 \mathrm{n}$.

\section{Commande des systèmes dynamiques hybrides : formalisation du problème}

Un système hybride est déterminé par le couplage d'une famille de systèmes dynamiques continus avec un automate. Il n'y a à un instant donné qu'un seul modèle continu actif: il correspond à un mode de fonctionnement du processus étudié. Le schéma suivant illustre cette structure 


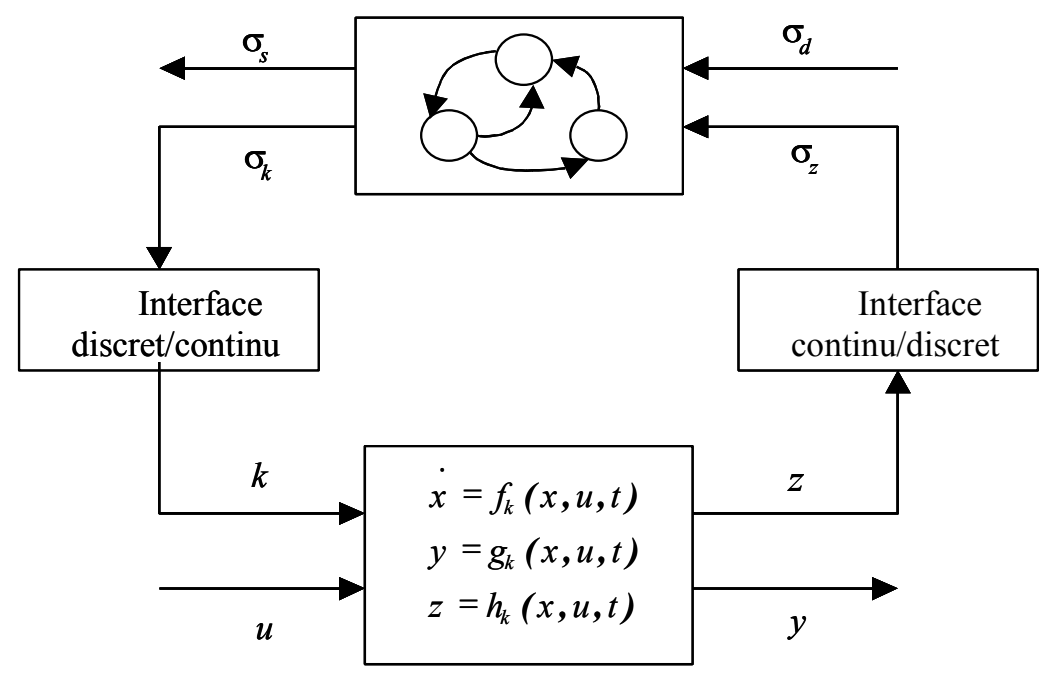

Nous supposons donc de manière très générale qu'un changement d'état discret $q$ est déterminé par la donnée d'une fonction de transition discrète :

$$
q(t)=v\left(q\left(t^{-}\right), x\left(t^{-}\right), d(t), t\right)
$$

où $d(t) \in D=\left\{d_{1}, d_{2}, \cdots, d_{m}\right\}$ représente une commande discrète. La fonction de transition continue pour un état discret $q$ est définie à partir de l'équation différentielle :

$$
\dot{x}=f_{q}\left(x(t), u_{q}(t), t\right)
$$

où $u_{q}(\cdot)$ désigne tout élément d'une classe de fonctions, défini sur un sous intervalle $T=\left[t_{0}, t_{f}\right]$ et prenant ses valeurs dans un domaine de commande $U_{q}$ attaché à l'état discret $q$. Le changement de valeur de $q$ provoqué par la commande discrète est appelé saut commandé

Pour concilier la dynamique discrète et continue, le temps est défini comme une variable continue et les variables discrètes, $q($.$) et d($.$) , sont considérées comme des$ fonctions constantes par morceaux. Par conséquent, la fonction de transition $v: Q \times X \times D \times T \rightarrow Q$ est continue par morceaux à droite. Cette mention est figurée par $t^{-}$dans [ 12]. Il est à noter que la condition « $q($.$) est constant par$ morceaux » implique un modèle «non zeno » c'est-à-dire un modèle qui ne tolère pas la possibilité d'une suite infinie de transitions en un temps fini.

Pour la clarté de l'exposé, on suppose que seules des discontinuités sur les champs de vecteurs sont modélisées. Nous renvoyons le lecteur à [RIE 99a] pour le cas général où des discontinuités sur le couple $(x, t)$ sont envisagées

L'image de $D$ par $v$ pour un état $(x(t), q(t))$ à un instant $t$ donné, est l'ensemble des modes disponibles. On le note $S(t)=v\left(q\left(t^{-}\right), x\left(t^{-}\right), D, t\right)$. Ce n'est pas un ensemble statique mais un ensemble constant par morceaux résultant de l'évolution de $x, q$ et du temps $t$. Une transition d'état $e=\left(q \rightarrow q^{\prime}\right)$ au point $(x, t)$ est dite contrainte si le passage en cette position $(x, t)$ modifie l'ensemble $S$ par suppression du mode $q$ ou adjonction du mode $q^{\prime}$. Nous supposerons que ces transitions résultent de la vérification par le couple $(x, t)$ de contraintes égalités, 
$C_{q, q^{\prime}}(x, t)=0$, correspondant à la frontière d'une région de l'espace $X \times T$ et dont le franchissement modifie l'ensemble $S$. Par exemple, pour un automate hybride, une garde Guard devient ou cesse d'être valide à l'instant $t$ (possibilité ou non d'un saut vers un état $q^{\prime}$ ) ou encore $x$ appartient à la frontière de l'invariant Inv (nécessité d'un saut par suppression de $q$ ). Ce cas est habituellement appelé saut autonome

En toute généralité, le problème de commande optimale considéré consiste à déterminer un couple de commande $(u, d)(\cdot)$ et une position initiale $s_{0}=\left(x_{0}, q_{0}\right) \in X_{0} \times Q_{0}$ tels que la fonction critère,

$$
J=\phi_{q\left(t_{f}\right)}\left(x\left(t_{f}\right), t_{f}\right)+\int_{t_{0}}^{t_{f}} L_{q(t)}(x(t), u(t), t) d t
$$

soit minimisée sous la contrainte finale $x\left(t_{f}\right) \in X_{f}$ où $X_{f}$ est un ensemble donné. La fonction $J$ est spécifiée par un problème concret, physique ou non, et représente par exemple une dépense d'énergie, d'argent, de temps, etc. L'instant initial $t_{0}$ est

donné et l'instant final $t_{f}$ peut être spécifié ou non. La fonction $\phi_{q}$ représente un critère terminal ou un coût à payer à l'arrivée.

\section{Application de la programmation dynamique}

Les premiers travaux sur la commande optimale qui s'inscrivent dans le cadre général des systèmes hybrides sont dus à Branicky

Nous donnons une adaptation du résultat original :

Théorème: Si une trajectoire admissible $(\mathrm{x}, \mathrm{q})($.$) déterminée par la donnée de la$ condition initiale $\left(\mathrm{x}_{0}, \mathrm{q}_{0}\right)$ et la commande $(\mathrm{u}, \mathrm{d})($.$) , est optimale alors les conditions$ suivantes sont vérifiées :

a) pour presque tout $\mathrm{t} \in\left[\mathrm{t}_{0}, \mathrm{t}_{\mathrm{f}}\right]$,

$$
\frac{\partial V(x, q, t, b)}{\partial t}=-\inf _{u}\left\{L_{q}(x, u, t)+\left[\frac{\partial V(x, q, t, b)}{\partial x}\right]^{T} f_{q}(x, u, t)\right\}
$$

b) pour presque tout $t$ et pour tout $\mathrm{d} \in \mathrm{D}$

$$
V(x(t), q(t), t, b) \leq V\left(x^{\prime}, q^{\prime}, t, b\right)
$$

avec

$$
\begin{gathered}
q^{\prime}=\varphi(x(t), q(t), d, t) \\
x^{\prime}=\phi(x, q, d, t)
\end{gathered}
$$


Différents algorithmes pour déterminer $V(x)$ sont exposés dans [BRA 95b]. Une première méthode consiste à transformer le problème sous la forme d'un problème de programmation linéaire en remplaçant les égalités ci-dessus par des inégalités. Le lien avec la commande impulsionnelle [BEN 84] est également souligné et un algorithme issu de ces systèmes est adapté.

Pour un problème simplifié ne comportant que des sauts commandés, S. Hedlund et A. Rantzer [HED 99] présentent une méthode de discrétisation dans $\mathfrak{R}^{2}$. L'approche basée sur un algorithme de programmation linéaire conduit à un problème d'optimisation convexe et l'estimation du critère est déterminée par une borne inférieure et supérieure. Deux exemples y sont présentés pour illustration.

\section{4-Le principe du maximum pour les $S D H$}

Une autre approche de la commande optimale des SDH peut être obtenue avec le Principe du Maximum de Pontryaguine [PON 64]. Ce principe permet pour un problème de commande classique (système continu) d'énoncer des conditions nécessaires vérifiées par la commande optimale. La prise en compte des discontinuités de modèles provoquées par les événements discrets est intégrée et garantit alors une optimisation globale du critère.

Définissons à présent pour chaque état discret $q$, la fonction Hamiltonienne associée :

$$
H_{q}\left(\lambda_{0}, \lambda, x, u, t\right)=\lambda^{T} f_{q}(x, u, t)-\lambda_{0} L_{q}(x, u, t)
$$

avec $\lambda_{0}$ une constante positive $\left(\lambda_{0} \geq 0\right)$ et le système Hamiltonien comme :

$$
\dot{x}=\frac{\partial H_{q}}{\partial \lambda} \quad \dot{\lambda}=-\frac{\partial H_{q}}{\partial x}
$$

Alors, on peut énoncer une version du principe du maximum pour un système hybride :

Théorème : $\mathrm{Si} \quad(u, d)(\cdot)$ et $(x, q)(\cdot)$ sont respectivement une commande admissible optimale et la trajectoire correspondante pour le problème [12]-[13] et [ 14], alors il existe une fonction vecteur absolument continue par morceaux $\lambda(\cdot)$ et une constante $\lambda_{0} \geq 0,\left(\lambda_{0}, \lambda\right) \neq(0,0)$ sur $T$, telles que :

(a) Le sextuplet $\left(\lambda_{0}, \lambda, x, q, u, d\right)(\cdot)$ satisfait le système Hamiltonien associé [ 16] presque partout

(b) Aux instants de commutation $\tau_{i}$, les conditions de transversalité suivantes sont satisfaites :

i. si $\tau_{i}$ est un instant de transition contraint sur une frontière définie par $p$ $\left(p<n=\operatorname{dim}\left(X_{q}\right)\right)$ contraintes égalités, $C_{q, q^{\prime}}(x, t)=0$, depuis le mode $q$ vers le mode $q$ ' alors : 


$$
\begin{aligned}
& \lambda\left(\tau_{i}^{+}\right)=\lambda\left(\tau_{i}^{-}\right)-\left.\frac{\partial C_{q, q^{\prime}}^{T}(x(t), t)}{\partial x} \pi\right|_{t=\tau_{i}} \\
& H_{q^{\prime}}\left(\lambda_{0}, \lambda, x, u, \tau_{i}^{+}\right)=H_{q}\left(\lambda_{0}, \lambda, x, u, \tau_{i}^{-}\right)+\left.\frac{\partial C_{q, q^{\prime}}^{T}(x(t), t)}{\partial t} \pi\right|_{t=\tau_{i}}
\end{aligned}
$$

avec $\pi$ un vecteur de dimension $\mathrm{p}$.

ii. si $\tau_{i}$ est un instant de transition non contraint depuis le mode $q$ vers le mode $q$ ' alors :

$$
\begin{aligned}
& \lambda\left(\tau_{i}^{+}\right)=\lambda\left(\tau_{i}^{-}\right) \\
& H_{q^{\prime}}\left(\lambda_{0}, \lambda, x, u, \tau_{i}^{+}\right)=H_{q}\left(\lambda_{0}, \lambda, x, u, \tau_{i}^{-}\right)
\end{aligned}
$$

(c) Pour un état généralisé $\left(\lambda_{0}, \lambda, x, q\right)(t)$ à un instant $t$ donné, la condition suivante de maximum sur le couple $(u, d)(t)$ est vérifiée si $t$ n'est pas un instant de commutation :

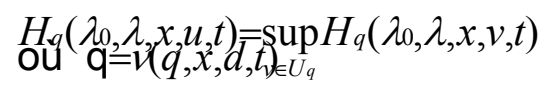

(d) $\lambda(\cdot)$ doit vérifier aux instants initial et final, les conditions de transversalité :

$$
\lambda\left(t_{0}\right) \in N_{C_{0}}\left(x\left(t_{0}\right)\right) \quad \lambda\left(t_{f}\right) \in N_{C_{f}}\left(x\left(t_{f}\right)\right)-\lambda_{0} \frac{\partial \phi_{q_{f}}\left(x\left(t_{f}\right), t_{f}\right)}{\partial x}
$$

où $N_{C}(x)$ représente le sous espace normal à $C$ au point $x$ (i.e. le sous-espace engendré par les vecteurs orthogonaux au plan tangent à $C$ au point $x) ; C_{0}$ et $C_{f}$ définissent respectivement les frontières des ensembles $X_{0}$ et $X_{f}$

Remarque : La constante $\lambda_{0}$ est très rarement égale à zéro et l'est dans des situations pathologiques. Aussi, dans la plupart des applications, on peut choisir $\lambda_{0}=1$.

Il s'agit d'un problème aux frontières. L'introduction du système [ 16], conduit à la résolution d'un système de $2 n$ équations pour lesquelles les conditions initiales et finales sont partagées aux instants initial et final. Les discontinuités de modèles s'accompagnent de conditions de raccordement sur la variable adjointe $\lambda$ exprimées par les conditions de transversalité [ 18]-[ 19]. La recherche de la commande discrète s'effectue comme en programmation dynamique avec des transitions d'états discrets contraintes par [ 18]-[19].

Lorsque les équations aux dérivées partielles d'HJB peuvent être définies, la variable adjointe $\lambda$ correspond à la dérivée partielle de la fonction de Bellman $V(x, t)$ par rapport à $x$ évaluée le long de la trajectoire optimale, soit $\lambda=-\lambda_{0} \frac{\partial V}{\partial x}$. De plus, il est facile de montrer que les équations d'HJB correspondent à $H=\lambda_{0} \frac{\partial V}{\partial t}$ 
le long de la trajectoire optimale. On voit donc que l'introduction du système adjoint permet d'établir une trajectoire et une commande optimale sans avoir à résoudre les équations d'HJB globalement. Cette approche a été éprouvée pour des problèmes de commande en temps optimal [RIE 99c] ainsi que pour la recherche d'une commande stabilisante par critère quadratique [RIE 99b]. Notons enfin qu'une méthode similaire basée sur une approche Lagrangienne, est développée dans [CEB 99].

\section{5- Exemple}

Considérons le système hybride défini par $\dot{x}=f(x)+u$ où la fonction à valeurs multiples $f(x)$ est représentée sur la figure $11 \mathrm{a}$. Ce système hybride présente très clairement des discontinuités de champs de vecteurs aux points intérieurs $\Delta$ et $-\Delta$. La nature de ces discontinuités est de type saut autonome. Un saut sur le champ $f$ se produit de la valeur 1 à la valeur -1 lorsque la variable d'état $x$ atteint par valeurs inférieures la frontière $\Delta$. Réciproquement, $f(x)$ passe de -1 à 1 lorsque la variable d'état $x$ atteint par valeurs supérieures la frontière $-\Delta$. On peut donc distinguer deux modes de fonctionnement $\dot{x}=f_{q}(x, u)$, pour $q=-1$ ou 1 avec $f_{q}(x, u)=q+u$.

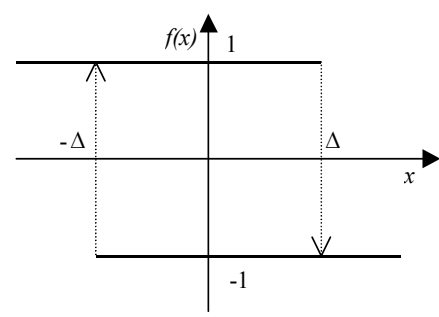

(a)

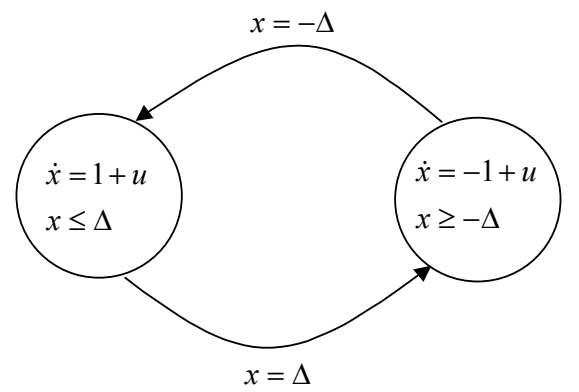

(b)

Figure 11:L'hystérésis (a) et l'automate associé (b)

La figure $11 \mathrm{~b}$ représente l'automate associé à la dynamique discrète de ce processus. Le problème de commande proposé est de minimiser,

$$
J=\frac{1}{2} \int_{0}^{\infty}\left(\alpha x^{2}+u^{2}\right) e^{-t} d t
$$

avec les conditions initiales $x(0)=x_{0}, q(0)=q_{0}$ et $u(t) \in \mathfrak{R}, \alpha \in \mathfrak{R}^{+}$.

\subsection{Résolution par le principe du maximum}

Cet exemple est résolu de manière analytique dans [RIE 99d] par le principe du maximum. La condition d'optimalité [ 19] conduit à $u=\lambda e^{t}$. A partir de [16], on établit entre deux instants de saut successifs $\tau_{k}$ et $\tau_{k+1}(k \in \mathfrak{R})$, le système [21]: 


$$
\left[\begin{array}{l}
\dot{x} \\
\dot{u}
\end{array}\right]=\left(\begin{array}{ll}
0 & 1 \\
q & 1
\end{array}\right)\left[\begin{array}{l}
x \\
u
\end{array}\right]+\left[\begin{array}{l}
q \\
0
\end{array}\right]
$$

La solution analytique de [ 21], pour $q=-1,1$ sur $\left[\tau_{k}, \tau_{k+1}\right.$ [, est donnée par :

$$
\begin{aligned}
& x_{q}(t)=\frac{q}{\alpha}+A_{k} e^{r_{1}\left(t-\tau_{k}\right)}+B_{k} e^{r_{2}\left(t-\tau_{k}\right)} \\
& u_{q}(t)=-q+r_{1} A_{k} e^{\eta_{1}\left(t-\tau_{k}\right)}+r_{2} B_{k} e^{r_{2}\left(t-\tau_{k}\right)}
\end{aligned}
$$

avec

$$
\begin{array}{ll}
r_{1}=\frac{1-\sqrt{1+4 \alpha}}{2}<0, \forall \alpha>0 & A_{k}=\frac{1}{r_{1} \sqrt{1+4 \alpha}}\left(q r_{2}-\alpha x_{q}\left(\tau_{k}\right)-r_{1} u_{q}\left(\tau_{k}\right)\right) \\
r_{2}=\frac{1+\sqrt{1+4 \alpha}}{2}>1, \forall \alpha>0 & B_{k}=\frac{1}{r_{2} \sqrt{1+4 \alpha}}\left(-q r_{1}+\alpha x_{q}\left(\tau_{k}\right)+r_{2} u_{q}\left(\tau_{k}\right)\right)
\end{array}
$$

Les conditions de transversalité [ 17] sur les frontières $C_{q}(x(t), t)=x-q \Delta=0$, $q=-1,1$ déterminent deux valeurs de saut possible sur la commande $u$ :

$$
\begin{aligned}
& u_{q}\left(\tau_{k}^{+}\right)=-u_{-q}\left(\tau_{k}^{-}\right) \\
& u_{q}\left(\tau_{k}^{+}\right)=u_{-q}\left(\tau_{k}^{-}\right)+2 q
\end{aligned}
$$

Par suite, la donné d'une condition initiale $\left(x_{0}, q_{0}, u_{0}\right)$ permet de générer l'ensemble des trajectoires candidates à l'optimalité. On montre alors que seuls deux comportements asymptotiques permettent d'obtenir un coût fini :

- soit le système converge après un nombre fini $\mathrm{k}$ de commutations vers le point $\lim _{t \rightarrow \infty}(x, u)=\left(\frac{q}{\alpha},-q\right)$ suivant le dernier mode $\mathrm{q}=-1,1$ emprunté ([ 22] avec $\left.B_{k}=0\right)$,

- soit $k=\infty$ et la solution est un cycle périodique autour de l'hystérésis.

La solution optimale est alors déterminée en évaluant le coût correspondant à chacune de ces situations. Cette méthode permet de mettre en évidence une bifurcation de la solution pour $\alpha>\alpha_{\max }=\frac{2+\sqrt{4+\Delta^{2}}}{\Delta^{2}} \approx 400.25$ (Figures. 12, 13a, et 13b). En effet, il s'avère que le comportement cyclique autour de l'hystérésis qui prévaut pour $\alpha$ inférieures à $\alpha_{\max }$, n'est plus optimal pour $\alpha>\alpha_{\max }$. Dans ce dernier cas, si le départ a lieu avec le mode $q$, la trajectoire $x$ converge sans commutation $(k=0)$ vers un point d'équilibre $\frac{q}{\alpha}$ et la commande $u$ converge vers la valeur $-q$ (cf. [22] avec $B_{0}=0$ ). 


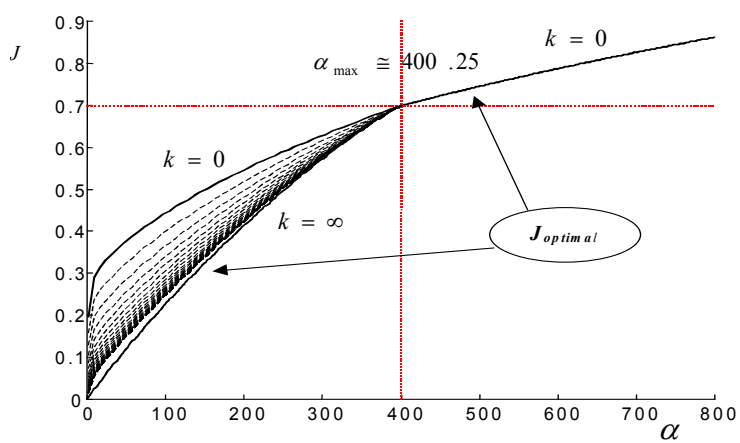

Figure 12: Evaluation du critère $J$ en fonction du nombre $k$ de commutations et du paramètre a pour $x_{0}=-0.2, \Delta=0.1$. Le critère optimal est déterminé par la courbe inférieure.

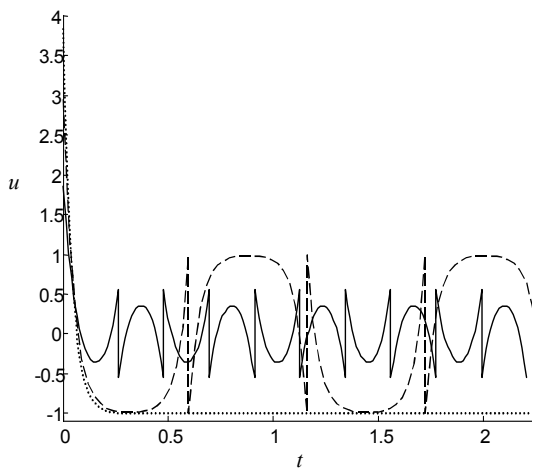

(a)

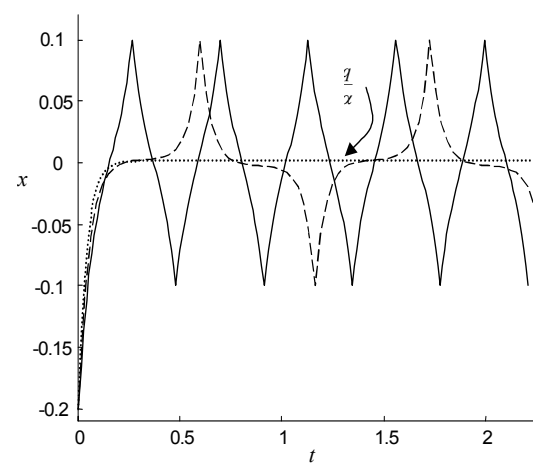

(b)

Figure 13: Commandes optimales (a) et trajectoires optimales (b) pour $\alpha=200$ (plein), $\alpha=400$ (pointillé), $\alpha=800$ (point), $x_{0}=-0.2, \Delta=0.1$

Lorsque $\alpha$ tend par valeurs inférieures vers $\alpha_{\max }$, on peut observer que (figures $12,13 a$ et $13 b)$ :

- la période du cycle augmente,

- la trajectoire se maintient alternativement (suivant le mode actif $q$ ) autour de la valeur $\frac{q}{\alpha}$,

- la limite correspond à une période infinie $\left(B_{0} \rightarrow 0\right)$.

On notera que l'ensemble des valeurs initiales pour la commande $u(0)=u_{0}$ menant à un coût fini, forme une suite de points isolés. Chacun de ces points correspond à une trajectoire obtenue pour un nombre fixé $k=0,1, \ldots$ de commutations. La suite converge vers une limite correspondant au régime périodique. Cette suite est réduite à un point lorsque $\alpha \geq \alpha_{\max } .5$ 


\subsection{Résolution par HJB et la programmation dynamique}

Branicky résout également ce problème numériquement dans [BRA 95b] à partir de l'algorithme suivant (problème aux frontières) :

1. Pour chaque $q$, fixer une valeur initiale à $V_{q}(x)$

2. Pour chaque $q$, pour $x \in A_{q}$ et $x \in C_{q}$, imposer les contraintes suivantes :

$$
\begin{aligned}
& V_{q}(x):=\min _{v, j}\left\{c_{a, q}(x, v)+e^{-\alpha \Delta_{a, q}(x, v)} V_{j}\left(G_{q}(x, v)\right)\right\} \\
& V_{q}(x):=V_{q}(x) \wedge \min _{z \in D, j}\left\{c_{c, q}(x, z)+e^{-\alpha \Delta_{c, q}(x, z)} V_{j}(z)\right\}
\end{aligned}
$$

3. Mettre à jour chaque $V_{q}(x)$ en résolvant séparément [ 8] sous la contrainte déterminée au point 2 .

4. Si la solution converge, reprendre depuis le point 2 .

Pour cet exemple, les délais et les coûts associés aux sauts autonomes sont nuls (soit $\Delta_{a, q}=c_{a, q}=0$ ) et les situations de saut commandé [25] ne doivent pas être prises en considération. Cet algorithme converge bien vers la solution optimale lorsque $\alpha$ est inférieure à $\alpha_{\max }$. En revanche, les solutions produites par Branicky au-delà de cette valeur ne sont plus optimales (non-détection de la bifurcation). Ce dernier point illustre bien les difficultés rencontrées dans la résolution numérique des problèmes de commande optimale hybride. La mise en place d'algorithmes appropriés (pour les deux méthodes) reste un problème ouvert.

\section{Conclusion}

L'utilisation de la programmation dynamique conduit automatiquement à la discrétisation des équations HJB. Une voie consiste alors à discrétiser le problème dès sa formulation. Des résultats intéressants sont obtenus dans le cas des systèmes pour lesquels les contraintes de saut définissent une partition de l'espace d'état et les équations différentielles associées à chaque mode sont linéaires.

Une approche qui nous paraît très prometteuse consiste à approcher la solution de l'équation de Bellman par encadrement.

L'utilisation du principe du maximum permet une caractérisation des solutions optimales. Elle peut sembler conduire à des résultats directement. En réalité se pose déjà la difficulté de résoudre un problème de tir comme dans le cas classique. Mais en plus, la dynamique discrète conduit à des bifurcations sur les trajectoires continues auxquelles sont associées des conditions de transversalité. Il est donc nécessaire d'explorer toutes les branches possibles comme dans la programmation dynamique. Cependant même si le nombre d'états discrets est fini, le nombre de branches peut lui être infini. 


\section{Références}

[BEN 84] Bensoussan A. et Lions J. L., Impulse Control and Quasi-Variational Equalities, Gauthier Villars, Paris 1984.

[BRA 95a] Branicky M. S., Studies in Hybrid Systems, Ph.D. dissertation, Dept. Elec. Eng. and Computer Sci., Massachusetts Inst. Technol., Cambridge, June 1995.

[BRA 95b] Branicky M. S. et Mitter S. K.,. Algorithms for Optimal Hybrid Control, Proceedings of the 34rd IEEE Conference on Decision and Control, New Orleans, LA- . (1995), p. 2661-2666.

[BRA 98] Branicky M. S., Borkar V.et Mitter S., A unified framework for hybrid control : Model and optimal control theory, IEEE Trans. on Automatic Control, Vol 43, p. 31-45, 1998.

[CEB 99] Cébron B., Sechilariu M.et Burger J., Optimal control of hybrid dynamical systems with hysteresis, Proceedings of European Control Conference, (1999).

[HED 99] Hedlund S.et Rantzer A., Optimal Control of Hybris Systems, Proceedings of. IEEE Conf. on Decision and Control, Phoenix, (1999).

[IQB 96] Iqbal N., Buisson J.et Quenec'hdu, Constrained Optimal Control of Descriptor systems : State feedback case, Symposium on Control, Optimization and Supervision, CESA'96, Lille (1996).

[KAL. ] KalmanR.E., Arbib et Falb Mathematical system theory, McGraw-Hill 1969

[LIO 82] Lions P. L. Generalized Solutions of Hamilton-Jacobi Equations, London Pittman, 1982.

[PON 64] Pontryagin L. P., Boltyanskii V. G., Gamkrelidze R. V. et Mishchenko E. F. The Mathematical Theory of Optimal Processes, Pergamon, 1964.

[RIE 99a] Riedinger P. Contribution à la commande optimale des systèmes dynamiques hybride, Thèse de l'Institut National Polytechniques de Lorraine (INPL), Nancy, décembre 1999.

[RIE 99b] Riedinger P., Kratz F., Iung C. et Zanne C. Linear Quadratic Optimization for Hybrid Systems, Proceedings of. IEEE Conf. on Decision and Control, Phoenix, (1999).

[RIE 99c] Riedinger P., Zanne C. et Kratz F. Time Optimal Control of Hybrid Systems, Proceedings of. American Control Conference, San Diego, (1999).

[RIE 99d] Riedinger P. et Iung C. Optimal Control for Hybrid Systems: An Hysteresis Example, Proceedings of.IEEE System, Man and Cybernetics, Tokyo, (1999).

[RIE 03] Riedinger P, Iung C., Kratz F. An Optimal Control Approach for Hybrid Systems EJC june 2003 